phases des tensions de ces réseaux peuvent s'ètablir indépendamment l'une de l'autre. Si les réscaux sont bouclés en plusieurs points, il laul employer un régulateur d'induction double. En choisissant alors convenablement la phase invariable de la tension réglable introduite par le régulateur d'induction double, on utilise cet appareil pour régler au point d'installation l'échange de puissance active ou de puissance réactive entre les deux réseaux. Si on place dans la ligne de liaison des réseaux deux survolteurs doubles en série, excités de façon à être le siège de tensions induites respectivement toujours en quadrature, on peut régler indépendamment l'échange de puissance active, par variation de la tension en quadrature avec la tension moyenne du réseau el l'échange de puissance réactive, par variation de la tension en phase avec la tension moyenne du réseau (1);

$4^{0}$ On intercale fréquemment des régulateurs d'induction du côté bagues des commutatrices dont on veut obtenir une tension du côté continu réglable dans de grandes limites. On sait que dans ce cas le réglage par bobine de self-induction el par variation du courant d'excitation ne peut guère dépasser $15 \%$, en raison soit du mauvais facteur de puissance sur le réseau altərnatif, soit du dimensionnement plus important imposé par l'échauffement pour certains organes de la commutatrice. Le régulateur d'induction s'emploie également avec des commutatrices à tension constante du côté contiuu, soit pour compenser les variations de tension du réseau alternatif, soit pour régler la charge de la commutatrice lorsque celle-ci fonctionne en parallèle avec d'autres machines. Dans le cas de distributions à trois fils dans lesquelles le fil neutre de côté continu est relié au point neutre du réseau alternalif (transformateur en général), le régulateur d'induction doit comporter autant d'enroulements qu'il y a de bagues du côté alternatif de la commutatrice, de façon à produir e

(1) Voir à se sujet le Bulletin de la Société Alsacienne de Constructions Mécaniques d'octobre 1925 et le compte rendu de la communication faite par $M$. Roth à la $1^{\text {re }}$ section de la Conférence Internationale des grands Réseaux de juin 1925. des variations symétriques et simultanées du potentiel de chaque bague par rapport à celui du point neutre.

$5^{\circ}$ Les grandes stations centrales possèdent aujourd'hui des alternateurs spéciaux pour l'essai et la mise sous tension progressive des câbles avant la connexion de ceux-ci aux rails d'alimentation. Il faut dans ce cas prévoir éventuellement les moyens de mise en phase nécessaires. Le régulateur d'induction peut également être utilisé dans ce cas; on le fait simple ou double selon qu'il doit servir uniquement à l'essai, ou également au couplage ; il n'y a pas alors de mise en phase à effectuer;

$6^{\circ}$ Dans les laboratoires, le régulateur d'induction rend également de grands services. Pour l'étalonnage de wattmètres ou de compteurs, on utilise le déphasage produit par le régulateur d'induction simple pour vérifier les indications de ces appareils sous différents facteurs de puissance, sans qu'il soit nécessaire de produire les charges correspondantes. Le régulateur d'induction simple permet en effet d'obtenir une résistance ohmique variable, par exemple en partant de la tension d'utilisation, un courant constant de phase variable par rapport à la tension d'alimentation (on alimente donc pour l'étalonnage le fil fin du wattmètre par la tension d'alimentation et on envoie le courant déphasé par rapport à cette tension dans le gros fil).

$7^{\circ}$ Dans les fours électriques, le régulateur d'induction monophasé ou polyphasé permet d'obtenir une tension variable, à partir d'une valeur sensiblement nulle si cela est nécessaire

Dans les usines modernes d'ammoniaque, on emploie également les régulateurs d'induction pour le réglage du chauffage des tubes de catalyse, etc...

Comme on le voit, les applications des régulateurs d'induction sont nombreuses et variées et ces appareils jouent un rôle important dans le transport et la distribution de l'énergie électrique.

\section{J. BERGER.}

\title{
Conditions de sécurité de la Traction électrique
}

On peut dire que la traction électrique des trains de grandes lignes est encore à son aurore, et qu'elle sera, dans l'avenir, l'objet de maint perfectionnement.

Une question, déjà agitée à plusieurs reprises, est celle de savoir s'il convient de confier à un seul homme la conduite d'une locomotive électrique, ou s'il est nécessaire de prévoir un mécanicien de rechange on tout an moins un aide, capable d'arrêter le convoi en cas de défaillance du titulaire. Avec beaucoup de raison on observe que le deuxième mécanicien n'aurait rien à faire, mais avec non moins de raison on observe que le titulaire peut être frappé de syncope et laisser entrevoir les terribles catastrophes qui pourraient survenir en pareil cas.

En tous pays, les mécaniciens de locomotive sont soumis à une sélection rigoureuse, résultant d'examens minutieux portant sur les connaissances techniques indispensables, puis sur la résistance physique, l'émotivité, la perfection de la vision, en particulier au point de vue du daltonisme, celte étrange infirmité qui fait confondre le rouge et le vert, précisément les couleurs des signaux. on peut dire que partout les mécaniciens sont des hommes d'élite ; toutefois, comme chacun de nous, ils peuvent ètre frappés de ces affections subites, parfois mortelles, la rupture d'anévrisme, la syncope, la congestion. Au point de vue accidents, ceux qui conduisent les locomotives électriques sont moins exposés que le conducteur d'une machine à vapeur et ils se trouvent dans des conditions de confortable bien supérieures. Placés dans un local fermé, ils sont complètement à l'abri des intempéries, et leur vue n'est pas gênée par la fumée et la vapeur, notamment dans les tunnels.

Quoique d'une autre nature, les accidents pouvant survenir à la machine même, et pouvant par contre-coup atteindre le mécanicien sont de mème importance sur la locomotive électrique et sur celle à vapeur. L'explosion de la chaudière à vapeur est très rare, et pour ma part je ne l'ai entendu qu'une fois, entre SaintLazare et le tunnel des Batignolles, à Paris, il y a une trentaine d'années. Il arrive plus fréquemment des ruptures de tubes, produisant une fuite de vapeur qui peut brûler le chauffeur au moment de l'ouverture de la porte du foyer. Sur la locomotive électrique peuvent se produire des courts-circuits, particulièrement redoutables par suite de la hạute tension employée. L'expé 
rience actuelle est encore trop courte pour qu'il ait été possible d'enregistrer des accidents de ce genre, au moins d'importance notable; cependant, il est probable que le cas se présentera un jour ou l'autre, lorsque ces engins, actuellement neufs, commenceront à ètre atteints par l'usure, due surtout aux trépidations. Dans quelle mesure en souffrira le mécanicien, c'est ce qu'il n'est pas possible de dire, pour le moment.

En résumé, l'expérience actuelle permel seulement de constater qu'un seul homme sur la locomotive est suffisant pour en assurer le service et que cet homme se trouve dans des conditions meilleures que le conducteur de l'appareil à vapeur. La présence d'un suppléant conduirait à une dépense considérable, qu'il convient d'éviter, tout en prenant certaines mesures que je vais exposer ci-après.

Le seul, mais grave danger que puisse faire courir la défaillance du mécanicien est que le train, privé de son conducteur, brûle les signaux, s'engage sur des voies obstruées ou vienne tamponner un autre convoi, et, à ce propos, je dois rappeler qu'un cas analogue a été envisagé pour les machines d'extraction des mines, où la question a été entièrement résolue par l'évite-molettes Reumaux, qui fonctionne de la façon suivante :

L'évite-molettes est constitué par un disque, solidaire du tambour d'enroulement des câbles qui supportent les cages, et muni de deux cames qui, lorsque l'une ou l'autre cage arrive à cinquante mètres du jour, ferment l'arrivée de la vapeur, ou coupent le courant électrique, suivant qu'il s'agit d'une commande à vapeur ou électrique. En cas de défaillance, ou même de simple inattention du mécanicien, la machine s'arrête, purement et simplement, et ne peut être remise en marche que par le rétablissement voulu de l'arrivée de vapeur ou de courant. Cet appareil, dû au regretté Elie Reumaux, ancien directeur des Mines de Lens, est universellement adopté dans les mines, où il a rendu d'inappréciables services.

Or, dans la traction électrique des chemins de fer, il serait facile d'introduire un dispositif semblable. Avant chaque station, le fil conducteur d'alimentation (fil de contact) devrait être coupé, c'est-à-dire devrait comprendre sur une certaine longueur une partie isolée. Sur la locomotive serait disposé un organe de déclanchement (disjoncteur) coupant le courant sur la machine même et provoquant le serrage des freins. La distance entre cette solution de continuité dans le fil de contact et la station serait calculée de telle sorte qu'un train de charge moyenne s'arrête automatiquement à l'endroit voulu. Tout accident serait donc évité en cas de défaillance du conducteur. La section de coupure devrait être très courte, juste assez longue pour produire le déclanchement du disjoncteur, mais dès son franchissement le train pourrait être prêt à repartir par la volonté du mécanicien. Un tel dispositif devrait être, bien entendu, installé devant chaque station, et lorsqu'il s'agirait de trains directs, le franchissement des stations intermédiaires serait assuré par le mécanicien luimême par le simple reclanchement du disjoncteur. Bien entendu, l'idée que je suggère est purement schématique et nécessite une étude de détail, notamment en ce qui concerne le fonctionnement intempestif des freins (dispositif de retardement, etc.).

A première vue je ne vois aucune objection à l'adoption de ce mode de protection, dont l'avenir justifiera peut-être l'opportunité.
On sait que différents Etats font actuellement des essais avec les trains électriques conduits par un seul homme et que cette innovation se heurte à la résistance obstinée du personnel. II s'agit d'un problème exclusivement technique. Si les essais sont concluants, si l'on arrive à établir que la sûreté des voyageurs est la même dans un train électrique conduit par un seul homme que dans un train dont la locomotrice est desservie par deux mécaniciens, on ne voit pas pourquoi les Compagnies de Chemins de fer renonceraient à une économie de cette importance.

Les appréciations sur ce problème que les chefs du personnel ferroviaire ont la fàcheuse, mais naturelle tendance d'envisager sous un angle politique, celui de la résistance à toute mesure pouvant amener une réduction du personnel et par conséquent une diminution de la puissance des syndicats dont ils sont les manœuvriers.

Les grandes lignes électriques du Loetschberg et des chemins de fer rhétiques (Suisse) font conduire depuis six ans déjà certains de leurs trains par un seul homme, sans qu'il soit arrivé aucun accident de ce fait.

La conduite d'une locomotive électrique est beaucoup plus simple que celle d'une locomotive à vapeur. La technique a réduil à un très petit nombre les appareils et les instruments que le mécanicien doit manipuler. Le conducteur, placé dans une cage de verre devant sa machine a une vue beaucoup plus nette et plus claire de la voie que le machiniste d'une locomotive à vapeur, incommodé par une longue chaudière, entourée de fumée et de vapeur. Le conducteur de la machine électrique ne manipule pas plus d'instruments que le wattman d'un tramway. Sans doute, sa machine marche beaucoup plus vite, mais elle roule sur des rails protégés par des barrières, tandis que le tramway parcourt des rues encombrées et a l'esprit sans cesse tendu pour éviter des obstacles.

Le conducteur de l'électromotrice ayant sa machine complète ment en main, voyant parfaitement la voie devant lui, n'ayant pas à se préoccuper de l'énergie qui arrive automatiquement, la présence d'un second machiniste à ses côtés est superflue. Ce second n'aurait pas d'autre occupation que d'observer le premier. En cas de défaillance du conducteur, la technique a prévu des appareils de sûreté qui entrent immédiatement en fonction lorsque la machine n'est plus conduite. La présence d'un second machiniste est plus nuisible qu'utile. La conduite d'une machine électrique est une chose trop absorbante pour pouvoir être faite par deux hommes dont ni l'un ni l'autre ne serait entièrement occupé.

Souvent des accidents auraient pu être évités si la machine avait été conduite par un seul homme. Lors d'un tamponnement récent d'un train et d'une automobile, l'enquête a prouvé qu'au moment de l'accident un second employé se trouvait, sans en avoir le droit, à côté du machiniste et s'entretenait avec lui, ce qui a été la cause de l'accident. A Bellinzona (Suisse), le train qui a dépassé un signal fermé était conduit par deux locomotives, montées par quatre hommes.

On conclut à l'inutilité de maintenir la conduite de deux hommes pour les catégories de trains actuellement desservis depuis des années par un seul homme sur d'autres lignes électriques, sans aucun accident. 